\title{
Choque térmico em filtros cerâmicos do sistema $\mathrm{Al}_{2} \mathrm{O}_{3}-\mathrm{SiC}$ (Thermal shock on ceramic filters in the system $\mathrm{Al}_{2} \mathrm{O}_{3}-\mathrm{SiC}$ )
}

\author{
V. R. Salvini, M. D. M. Innocentini, V. C. Pandolfelli \\ Departamento de Engenharia de Materiais - DEMa \\ Universidade Federal de S. Carlos - UFSCar \\ Rod.Washington Luiz, km 235, CP 676, 13565-905, S. Carlos, SP \\ pvrs@iris.ufscar.br,vicpando@power.ufscar.br
}

\begin{abstract}
Resumo
Em diversas aplicações as cerâmicas celulares são submetidas a tensões térmicas severas, tal como na filtração de metais fundidos. Contudo, há poucos estudos sobre o desempenho ao choque térmico destes materiais. Uma das razões é que a metodologia para análise desta classe de materiais deve ser distinta daquela utilizada para os materiais cerâmicos densos. Isto porque no caso dos materiais celulares para filtração de metais fundidos o meio causador do choque térmico infiltra-se rapidamente na estrutura reticular de poros, reduzindo o gradiente de temperatura entre a superfície externa e a do interior do corpo. Neste contexto, a proposta do presente trabalho é verificar os efeitos de algumas propriedades dos filtros cerâmicos em seu comportamento mecânico durante testes de choque térmico em água. As propriedades consideradas são a permeabilidade, a condutividade térmica e a área superficial específica dos filtros. Para isto foram utilizados os filtros cerâmicos do sistema de $\mathrm{Al} \mathrm{O}_{3}-\mathrm{SiC}$ de $8,10,20$ e 30 ppi (poros por polegada linear).
\end{abstract}

Palavras-chave: filtro cerâmico, choque térmico, permeabilidade.

\begin{abstract}
In many applications, open-cell ceramics are expected to undergo severe thermal stresses, for instance, in their use as molten metal filters. However, only a few studies have considered the thermal shock behavior of these materials. One of the main reasons is the theoretical approach used for dense ceramics which may not be valid for porous materials. In this context, the aim of this work is to analyze the effects of permeability, specific surface area and thermal conductivity on the mechanical behavior of ceramic filters subjected to water quenching tests. Al O -SiC filters with nominal cell sizes, expressed as the number of pores per linear inch (ppi), ranged from 8 to 30 ppi were used in the experimental tests.
\end{abstract}

Keywords: ceramic filter, thermal shock, permeability.

\section{INTRODUÇÃO}

Os estudos sobre choque térmico de filtros cerâmicos encontrados na literatura [1-4] baseiam-se na análise termoelástica das tensões térmicas para o início de fratura estabelecida por Kingery [5].

Segundo Kingery [5], a tensão térmica $\left(\sigma_{\mathrm{T}}\right)$ gerada em um corpo denso biaxialmente engastado resfriado rapidamente pode ser expressa pela seguinte equação:

$$
\sigma_{\mathrm{T}}=\frac{\mathrm{E} \alpha \Delta \mathrm{T}}{(1-\mathrm{v})}
$$

onde $\Delta \mathrm{T}$ é a diferença de temperatura entre a superfície (Ts) e o centro do corpo (Tc); $\alpha$, o coeficiente de expansão térmica linear; E, o módulo elástico e v, a razão de Poisson. Quando a tensão de tração gerada for igual a de fratura do material $\left(\sigma_{\mathrm{T}}=\sigma_{\mathrm{F}}\right)$, dá-se início à propagação de trinca.

Estudos complementares de choque térmico [5-6] têm indicado a necessidade de se incluir na equação (A) as condições térmicas dependentes do tempo. Assim, foi introduzido nesta equação um fator de atenuação da tensão térmica $\psi$, resultando na expressão:

$$
\sigma_{\mathrm{T}}=\frac{\psi \mathrm{E} \alpha \Delta \mathrm{T}}{(1-\mathrm{v})}
$$

O parâmetro $\psi$ é calculado à partir do módulo de Biot $(\beta)$ pela seguinte equação:

$$
\psi^{-1}=1,5+\frac{3,25}{\beta}-0,5 \exp \left(-\frac{16}{\beta}\right)
$$

sendo o módulo de Biot $(\beta)$ dado por:

$$
\beta=\frac{\mathrm{r}_{\mathrm{m}} \mathrm{h}}{\mathrm{K}}
$$

onde $r_{m}$ é o comprimento característico do material na direção de maior troca de calor, h é o coeficiente de transferência de calor entre o fluido e o material e $\mathrm{K}$, a condutividade térmica do material.

Rearranjando a equação (B) tem-se:

$$
\Delta \mathrm{T} \mathrm{c}=\mathrm{R}=\frac{\sigma_{\mathrm{T}}(1-\mathrm{v})}{\psi \mathrm{E} \alpha}
$$

O parâmetro R representa os resultados da análise termoelástica destacando-se o critério de máxima tensão para início de fratura. A dimensão do parâmetro $\mathrm{R}$ é expressa em $\left({ }^{\circ} \mathrm{C}\right)$ e corresponde à menor 
diferença de temperatura necessária para gerar uma tensão térmica de intensidade suficiente para propagar uma trinca através do material. A esta diferença de temperatura dá-se o nome de diferença de temperatura crítica, $\Delta \mathrm{T}_{\mathrm{C}}$.

Fazendo uso destes conceitos, foi desenvolvida uma equação para avaliar a resistência ao choque térmico de filtros cerâmicos [2]. Para isto, foi considerado que o material sólido dos filamentos tem uma resistência ao choque térmico expressa por $\mathrm{R}_{\mathrm{s}}$, a qual pode ser calculada substituindo-se na equação (E) as expressões de módulo elástico e de tensão de fratura desenvolvidas para materiais de células abertas [2] como dadas a seguir:

$$
\mathrm{E} \approx \mathrm{C}_{1} \mathrm{E}_{\mathrm{s}}\left(\frac{\rho}{\rho \mathrm{s}}\right)^{2}
$$

e

$$
\sigma_{\mathrm{F}} \approx \mathrm{C}_{2} \sigma_{\mathrm{s}}\left(\frac{\rho}{\rho \mathrm{s}}\right)^{3 / 2}
$$

onde $\mathrm{E}_{\mathrm{S}}$ é o módulo elástico do material sólido dos filamentos, $\mathrm{E}$ o módulo elástico do filtro, $\sigma_{\mathrm{F}}$ a tensão de fratura do filtro sob compressão, $\sigma_{\mathrm{S}}$ a tensão de fratura do material sólido dos filamentos sob flexão, $\mathrm{C}_{1}$ e $\mathrm{C}_{2}$ são constantes iguais a 0,50 e 0,16 , respectivamente, e $\left(\rho / \rho_{\mathrm{s}}\right)$ a densidade relativa da estrutura celular, sendo $\rho$ a densidade geométrica da estrutura e $\rho_{\mathrm{s}}$ a densidade real do material que constitui os filamentos sólidos.

Substituindo-se (F) e (G) na equação (E) e assumindo $\psi=1$ (máxima severidade de choque térmico onde $\beta \rightarrow \infty$ ), chega-se à seguinte expressão [2]:

$$
\mathrm{R}_{\mathrm{F}}=\mathrm{R}_{\mathrm{s}}\left(\frac{\mathrm{C}_{2}}{\mathrm{C}_{1}}\right)\left(\frac{\rho}{\rho \mathrm{s}}\right)^{-1 / 2}
$$

onde $\mathrm{R}_{\mathrm{F}}$ é o parâmetro de resistência ao choque térmico do filtro e $\mathrm{Rs}=\frac{\sigma_{\mathrm{s}}(1-\mathrm{v})}{\mathrm{E}_{\mathrm{s}} \alpha_{\mathrm{s}}}$ é a equação (E) para o sólido do filtro.

A equação $(\mathrm{H})$ indica que a resistência ao choque térmico de filtros diminui à medida que a densidade relativa dos mesmos aumenta [2].

Estudando o comportamento de choque térmico de filtros cerâmicos de $\mathrm{Al}_{2} \mathrm{O}_{3}$ de 7, 20 e 65 ppi no intervalo de temperatura entre 25 e $700^{\circ} \mathrm{C}$, foi observada experimentalmente uma degradação progressiva das propriedades mecânicas dos filtros, ao invés de um $\Delta \mathrm{T}_{\mathrm{C}}$ característico [3]. No entanto, aplicando-se na equação $(\mathrm{H})$ os valores das propriedades dos filtros produzidos, foi obtido $\mathrm{R}_{\mathrm{F}} \sim 140^{\circ} \mathrm{C}$. Foi também observado que o dano variou com o tamanho de poro dos filtros.

A não aplicabilidade da equação $(\mathrm{H})$ aos dados experimentais de choque térmico de filtros cerâmicos pode ser atribuída a inexistência de uma determinação correta do parâmetro de atenuação $\psi$ para cálculo do $\mathrm{R}_{\mathrm{S}}$ [3]. Foi ainda sugerido que para o cálculo de $\psi$ deveria ser considerada a permeabilidade dos filtros, porém não foi estudada esta propriedade.

A obtenção do valor correto de $\psi$ depende do cálculo do módulo de Biot, conforme a equação C. Mas, devido à dificuldade de se determinar o coeficiente de transferência de calor (h) para choque térmico de cerâmicas em água [7], adota-se na literatura a condição mais severa de choque térmico onde $\beta>20$ e $\psi \cong 1$. Porém, acredita- se que para filtros cerâmicos tal condição não é a mais adequada. Além disso, a equação (H) de Gibson e Ashby não valida os dados experimentais de choque térmico de filtros cerâmicos porque ela explica somente o que acontece com o filamento e não o comportamento geral do dano no filtro como um todo.

Foi também observado dano mecânico progressivo durante os testes de choque térmico de filtros de $\mathrm{SiC}$, YZA e AZ, ao invés de um valor característico de $\mathrm{R}_{\mathrm{F}}$ [4]. Contudo, foi proposto que o parâmetro $\mathrm{R}_{\mathrm{F}}$, , que considera a condutividade térmica do filtro $\left(\mathrm{K}_{\mathrm{T}}\right)$, seria mais adequado para se avaliar o comportamento de choque térmico de filtros cerâmicos. Porém, ao avaliar o comportamento de $\mathrm{R}_{\mathrm{F}}$ ' em função dos resultados experimentais, foi observado que este parâmetro também não explica o comportamento de choque térmico de filtros cerâmicos.

Até o momento, observa-se nos estudos de choque térmico de filtros cerâmicos [2-4] a adoção dos parâmetros de resistência ao choque térmico $\left(R_{F}, R_{F}{ }^{\prime}\right)$ na tentativa de explicar, sem sucesso, o comportamento experimental observado para estes materiais. Mas, segundo a literatura [1, 5-7], estes parâmetros governam somente o mecanismo de nucleação e início de propagação de trincas em materiais cerâmicos densos de elevada resistência mecânica e baixa porosidade. Portanto, para materiais que contém microtrincas e elevada porosidade, tal como os filtros cerâmicos, a preocupação deve ser quanto aos parâmetros que afetam a propagação destas trincas causando dano mecânico ao corpo cerâmico [1]. Assim, acredita-se que o estudo de choque térmico de filtros cerâmicos deve ser baseado na minimização do dano e não em se evitar o início de propagação de trinca.

Dentro deste contexto, a proposta do presente trabalho é verificar os efeitos de algumas propriedades dos filtros cerâmicos em seu comportamento mecânico durante testes de choque térmico em água. As propriedades consideradas são a permeabilidade, a condutividade térmica e a área superficial específica dos filtros. Para isto foram utilizados os filtros cerâmicos do sistema de $\mathrm{Al}_{2} \mathrm{O}_{3}$ $\mathrm{SiC}$ de 8, 10, 20 e 30 ppi.

\section{MATERIAIS E MÉTODOS}

Os filtros foram produzidos pelo método da réplica [8]. As esponjas poliméricas foram impregnadas com uma suspensão cerâmica aquosa contendo de $\mathrm{Al}_{2} \mathrm{O}_{3}$ e $\mathrm{SiC}$ (relação 1:1 em volume) e aditivos para adequar as condições de processamento. Após a secagem a $100{ }^{\circ} \mathrm{C} / 24$ h e calcinação a $900^{\circ} \mathrm{C} / 2 \mathrm{~h}$ para a eliminação da água e dos orgânicos, respectivamente, os filtros foram sinterizados a $1250{ }^{\circ} \mathrm{C}$ por 5 horas ao ar [9].

Caracterização física. A fração volumétrica do espaço vazio no filtro determina sua porosidade $(\varepsilon)$, expressa por $\left[1-\left(\rho / \rho_{s}\right)\right]$. A densidade geométrica $(\rho)$ foi medida a partir das dimensões e massa dos corpos-de-prova e, a densidade real $\left(\rho_{\mathrm{s}}\right)$, foi determinada por picnometria de hélio (Accupyc - Micromeritics) dos filamentos moídos. $\mathrm{O}$ diâmetro das células foi determinado por análise de imagens e os aspectos microestruturais foram avaliados por microscopia eletrônica de varredura (MEV) no Lyca Stereoscan 440. A partir dos dados de porosidade ( $\varepsilon$ ) e tamanho de poro (d) calculou-se, pelo método de Richardson et. al. [10], a área superficial por unidade de volume $\left(\mathrm{S}_{\mathrm{v}}\right)$ dos filtros. Segundo a literatura $[2,12]$, o modelo geométrico que melhor representa a estrutura reticulada é o tetradecaedro, sendo a célula unitária deste constituída por 14 faces ( 8 hexágonos e 6 quadrados) interligadas por filamentos 
triangulares. A seqüiência de cálculo utilizada para determinação de $S_{\mathrm{v}}$ foi:

a) cálculo do comprimento do filamento do hexágono (I) :

I = 0,5498d/[ $\left.1-0,971(1-\varepsilon)^{0,5}\right]$

b) cálculo da espessura do filamento $\left(\mathrm{t}_{\mathrm{s}}\right): \mathrm{t}_{\mathrm{s}}=0,971(1-\varepsilon)^{0,5}$ |

c) cálculo do volume da célula $\left(\mathrm{V}_{\mathrm{c}}\right): \mathrm{V}_{\mathrm{c}}=11,31 \mathrm{I}^{3}$

d) cálculo da área superficial dos filamentos $\left(\mathrm{S}_{\mathrm{s}}\right): \mathrm{S}_{\mathrm{s}}=36 \mathrm{t}_{\mathrm{s}} \mathrm{I}$

e) cálculo da área superficial por unidade de volume $\left(\mathrm{S}_{\mathrm{v}}\right)$ :

$\mathrm{S}_{\mathrm{v}}=\mathrm{S}_{\mathrm{s}} /\left[\mathrm{V}_{\mathrm{c}}(1-\varepsilon)\right]$

Caracterização fluido dinâmica. Após a caracterização física, os filtros foram analisados quanto à permeabilidade em um equipamento hidrodinâmico desenvolvido em laboratório [9]. As constantes de permeabilidade Darciana $\left(\mathrm{k}_{1}\right)$ e não-Darciana $\left(\mathrm{k}_{2}\right)$ foram determinadas pela equação de Forchheimer para fluidos incompressíveis:

$$
\frac{\Delta \mathrm{P}}{\mathrm{L}}=\frac{\mu}{\mathrm{k}_{1}} \mathrm{v}_{\mathrm{s}}+\frac{\rho_{\mathrm{f}}}{\mathrm{k}_{2}} \mathrm{v}_{\mathrm{s}}^{2}
$$

onde $\Delta \mathrm{P}$ é a queda pressão através do filtro; $\mathrm{L}$ é a espessura do filtro; $\mu$ e $\rho_{\mathrm{f}}$ são, respectivamente, a viscosidade e a densidade do fluido; $v_{s}$, a velocidade superficial do fluido obtida pela razão entre a vazão volumétrica e a área da secção transversal da amostra ortogonal ao escoamento. Os termos da equação (I) apresentam as contribuições viscosas (Darciana) e inerciais (não-Darciana) à queda de pressão total do fluido. A predominância de um ou outro termo na queda de pressão para um dado fluido é função da velocidade utilizada e das constantes $\mathrm{k}_{1}$ e $\mathrm{k}_{2}$ [11].

Caracterização térmica. A condutividade térmica total dos filtros $\left(\mathrm{K}_{\mathrm{T}}\right)$ foi calculada utilizando-se o modelo proposto [12] para esponjas e adaptado para materiais celulares de células abertas [2]. Calculou-se a condutividade térmica na faixa de temperatura de $25^{\circ} \mathrm{C}$ a $900{ }^{\circ} \mathrm{C}$, considerando-se os dados obtidos de tamanho de poro (d), densidade relativa $\left(\rho / \rho_{\mathrm{s}}\right)$, espessura da amostra $(\mathrm{L})$ e da proporção volumétrica de $\mathrm{Al}_{2} \mathrm{O}_{3}\left(\mathrm{~V}_{\mathrm{d}}\right)$ e de $\mathrm{SiC}\left(\mathrm{V}_{\mathrm{m}}\right)$ na composição dos filtros. Segundo $[2,12]$, a condutividade térmica total do filtro $\left(\mathrm{K}_{\mathrm{T}}\right)$ corresponde à soma das contribuições da condutividade do filtro a baixas temperaturas onde não há radiação $\left(\mathrm{K}_{\mathrm{F}}\right)$ e da condutividade por radiação $\left(\mathrm{K}_{\mathrm{r}}\right)$. Utilizou-se a seguinte sequiência de cálculo:

a) condutividade da parte sólida $\left(\mathrm{K}_{\mathrm{S}}\right): \mathrm{K}_{\mathrm{s}}=1 / 3 . \mathrm{K}_{\text {composição }} \cdot\left(\rho / \rho_{\mathrm{s}}\right)$ b) condutividade do filtro a baixas temperaturas $\left(\mathrm{K}_{\mathrm{F}}\right)$ :

$\mathrm{K}_{\mathrm{F}}=\mathrm{K}_{\mathrm{S}} \cdot(\mathrm{d} / \mathrm{L}) \cdot\left(\rho / \rho_{\mathrm{s}}\right)$

c) condutividade por radiação $\left(\mathrm{K}_{\mathrm{r}}\right): \mathrm{Kr}=4 \vartheta \Omega \overline{\mathrm{T}}^{3} \mathrm{~L} \cdot \exp \left[-\mathrm{e}_{\mathrm{s}} \cdot\left(\rho / \rho_{\mathrm{s}}\right) \cdot \mathrm{L}\right]$ sendo $\vartheta$, a emissividade da superfície; $\Omega$, a constante de Stefan Boltzmann e $\overline{\mathrm{T}}$, a temperatura média.

d) condutividade térmica total do filtro $\left(\mathrm{K}_{\mathrm{T}}\right)$ : $\mathrm{K}_{\mathrm{T}}=\mathrm{K}_{\mathrm{F}}+\mathrm{K}_{\mathrm{R}}$

Caracterização termomecânica. Os ensaios de choque térmico dos filtros em água foram realizados em um forno vertical Lindberg Blue M. A amostra do filtro foi suspensa dentro do forno por um fio de kanthal. Paralelo ao corpo cerâmico tinha-se um termopar cromel-alumel para determinar a temperatura instantânea da amostra $\left(\mathrm{T}_{\text {amostra }}\right)$. Uma vez atingida a temperatura de teste, a amostra permaneceu por mais 30 minutos dentro do forno e, em seguida, o fio de kanthal foi solto e o conjunto amostra-fio foi mergulhado em um tanque com água. Deste modo, a temperatura de choque térmico na amostra correspondeu à diferença entre a temperatura da amostra e da água $\left(\Delta \mathrm{T}=\mathrm{T}_{\text {amostra }}-\mathrm{T}_{\text {água }}\right)$. Para cada temperatura de teste foram ensaiadas 4 amostras.
Após o ensaio de choque térmico as amostras foram secas em estufa a $100{ }^{\circ} \mathrm{C} / 24 \mathrm{~h}$ e, em seguida, ensaiadas quanto à resistência mecânica sob compressão em uma máquina MTS 810 com velocidade do atuador de $1,3 \mathrm{~mm} / \mathrm{min}$. As superfícies da amostra ortogonais à aplicação da carga de compressão foram cobertas com uma borracha semi-rígida. Isso é recomendado para eliminar os efeitos de carregamento localizado, devido à topografia da superfície das amostras [9].

\section{RESULTADOS E DISCUSSÃO}

Geralmente, o dano causado por choque térmico em materiais cerâmicos é avaliado pela redução do valor da resistência mecânica. A Fig. 1 mostra a variação da resistência mecânica sob compressão dos filtros estudados em função da temperatura de choque térmico. Da mesma forma observada anteriormente [3, 4], verifica-se nesta figura uma redução gradual da resistência mecânica dos filtros à medida que a temperatura de choque térmico aumenta, indicando, assim, um processo de dano mecânico progressivo ao invés da propagação catastrófica de trinca. Cabe mencionar que não se observou a desintegração total das amostras durante os testes, mesmo quando estas foram submetidas a $\Delta \mathrm{T}=900{ }^{\circ} \mathrm{C}$.

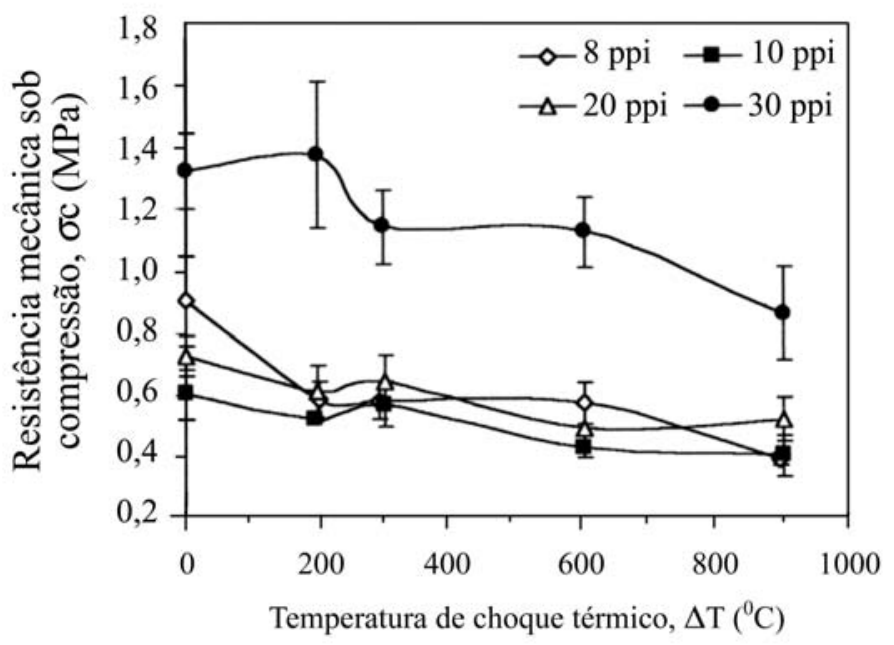

Figura 1: Resistência mecânica sob compressão em função da temperatura de choque térmico em água.

[Figure 1: Crushing strength as a function of thermal shock temperature in water.]

A análise por microscopia eletrônica de varredura (MEV) das amostras antes e após o choque térmico indica que o processo de dano mecânico é causado pela propagação das trincas pré-existentes nos filamentos. Estas trincas são geradas na etapa de processamento dos filtros, especificamente durante a eliminação da esponja polimérica [13]. Na Fig. 2 apresenta-se a estrutura macroscópica do filtro de 8 ppi antes e após o choque térmico.

Ainda quanto à morfologia do dano observado por MEV, em nenhuma das amostras analisadas observou-se a propagação de uma única trinca atravessando várias células do filtro, mesmo quando submetido ao choque térmico de $900^{\circ} \mathrm{C}$. Desse modo, pode-se supor que, embora o gradiente de temperatura seja através do filtro como um todo ( 'bulk"), o dano estrutural causado no filtro está relacionado à tensão térmica que atua nos filamentos individualmente [2-4].

Uma vez que para estes materiais não se observou 


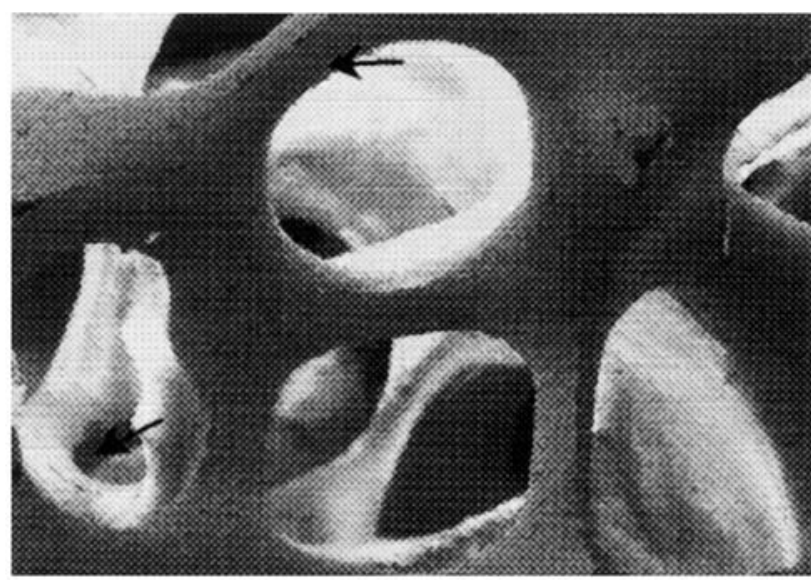

(a)

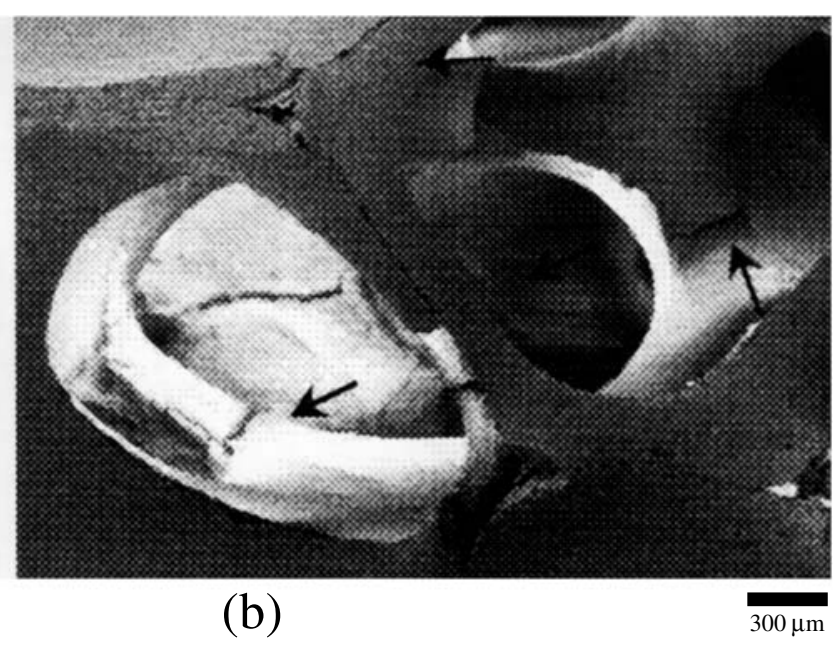

(b)

Figura 2: Microscopia eletrônica de varredura do filtro de 8 ppi: (a) antes e (b) após choque térmico de $900{ }^{\circ} \mathrm{C}$. As setas indicam as trincas nos filamentos.

[Figure 2: Scanning electron microscopy of 8 ppi filter: (a) before and (b) after thermal shock of $900{ }^{\circ} \mathrm{C}$. The arrows indicate microcracks on the strut surface.]

experimentalmente um $\Delta \mathrm{T}_{\mathrm{c}}$ característico, ou seja, uma variação de temperatura crítica na qual tem-se a redução abrupta da resistência mecânica, adotou-se neste trabalho um parâmetro que representa o dano por choque térmico, $\mathrm{D}$, assim definido [5, 7]:

$$
\mathrm{D}=1-\frac{\sigma_{\mathrm{c}}}{\sigma_{0}}
$$

onde $\sigma_{\mathrm{C}}$ é a resistência mecânica sob compressão do filtro após choque térmico e $\sigma_{0}$, a resistência mecânica antes do choque térmico. Quanto maior o valor do parâmetro D, maior o dano mecânico causado na estrutura do material.

Na Fig. 3 apresenta-se o gráfico do parâmetro de dano por choque térmico (D) em função da temperatura de choque térmico $(\Delta \mathrm{T})$ para os filtros de $\mathrm{Al}_{2} \mathrm{O}_{3}-\mathrm{SiC}$ estudados.

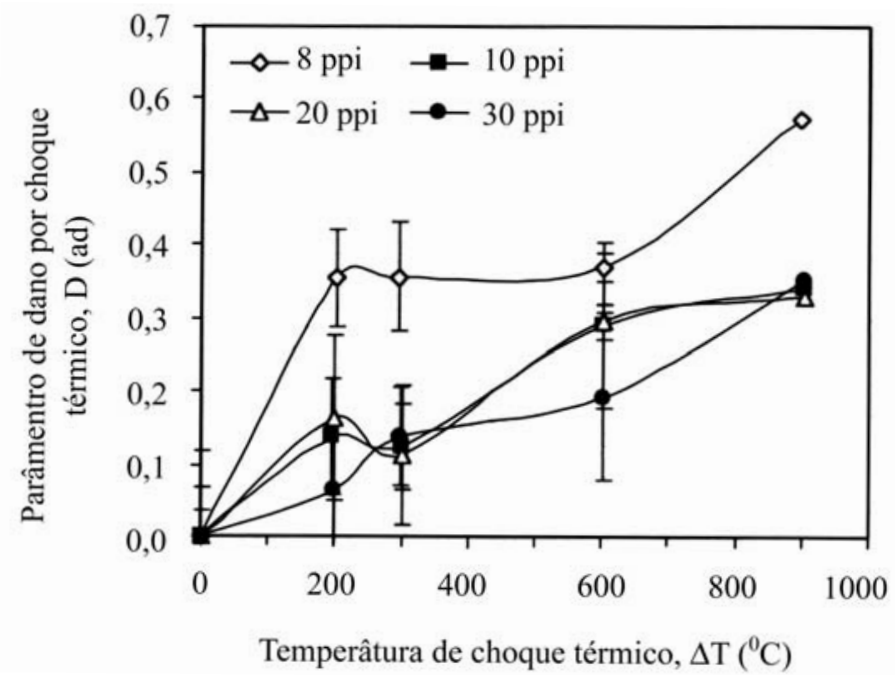

Figura 3: Comportamento do dano, $\mathrm{D}$, em função da temperatura de choque térmico, $\Delta \mathrm{T}$, para os filtros de $\mathrm{Al}_{2} \mathrm{O}_{3}-\mathrm{SiC}$.

[Figure 3: Behavior of the damage parameter, D, with thermal shock temperature, $\Delta T$, for $\mathrm{Al}_{2} \mathrm{O}_{3}$-SiC filters.]
A análise desta figura revela dois efeitos sobre o parâmetro de dano:

- efeito da temperatura de choque térmico $(\Delta \mathrm{T})$ : quanto maior a severidade de choque térmico maior a deterioração mecânica do filtro e

- efeito do tamanho de poro (célula): o filtro com maior tamanho de poro (8 ppi) apresentou os maiores valores de dano dentre todos os filtros analisados em toda a faixa de $\Delta \mathrm{T}$ analisada.

A dependência do parâmetro de dano D com o tamanho de poro é confirmada na Fig. 4, onde são plotados os valores de D após choque térmico de $900{ }^{\circ} \mathrm{C}$ em função do tamanho de poro. Neste gráfico escolheu-se o parâmetro $\mathrm{D}$ após $\Delta \mathrm{T}=900^{\circ} \mathrm{C}$ por representar a condição mais severa de choque térmico.

Observa-se na Fig. 4 que o dano apenas apresenta um valor diferenciado para a amostra de filtro de 8 ppi. O aumento do tamanho de poro influencia uma série de propriedades dos filtros, as quais

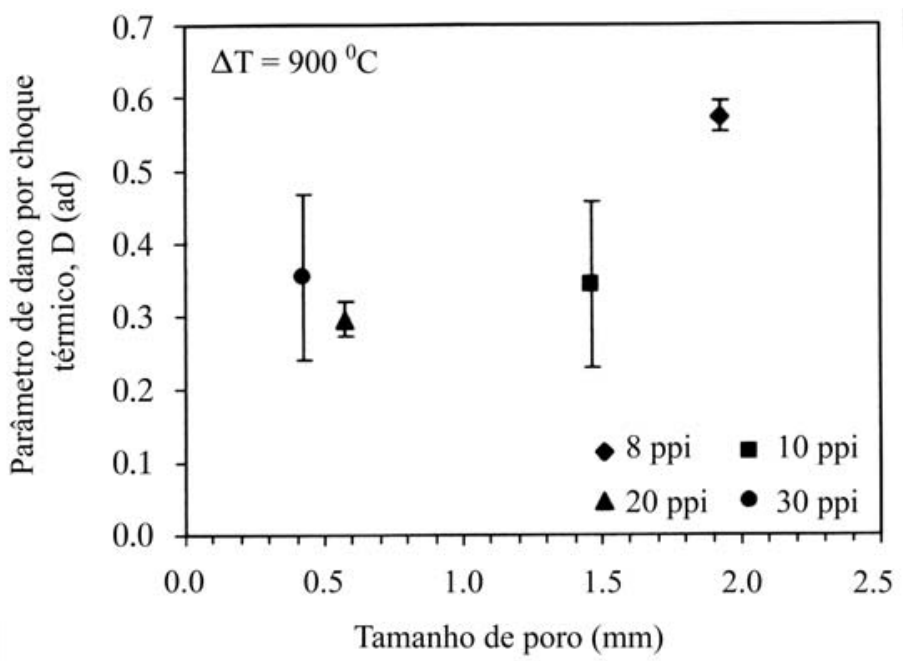

Figura 4: Dano por choque térmico, D, em função do tamanho de poro do filtro.

[Figure 4: Damage parameter, D, as a function of filter pore size.] 
estariam atuando no comportamento de choque térmico.

Neste contexto, faz-se a seguir uma análise do comportamento da permeabilidade, área superficial por unidade de volume e condutividade térmica em função do tamanho de poro dos filtros de $8,10,20$ e 30 ppi.

$\mathrm{O}$ efeito do tamanho de poro dos filtros na permeabilidade Darciana $\left(\mathrm{k}_{1}\right)$ e não-Darciana $\left(\mathrm{k}_{2}\right)$ é ilustrado na Fig. 5. Verifica-se que tanto no regime viscoso $\left(\mathrm{k}_{1}\right)$ quanto no inercial $\left(\mathrm{k}_{2}\right)$ a permeabilidade aumenta a medida que o tamanho de poro cresce, concordando com resultados anteriores [11].

Para entender o efeito da permeabilidade no comportamento de choque térmico de filtros cerâmicos, deve-se fazer algumas considerações sobre as propriedades fluidodinâmicas destes materiais.

Sabe-se que quanto maior a permeabilidade da estrutura de poros mais fácil será o acesso do fluido frio até os filamentos localizados no interior do filtro. Assim, pode-se assumir que tanto os filamentos da superfície externa como os localizados no interior do filtro sofrerão choque térmico $(\Delta \mathrm{T})$ elevado e de mesma intensidade, uma vez que $\Delta \mathrm{T}=\mathrm{T}_{\text {filamento }}-\mathrm{T}_{\text {água fria }}$. Consequentemente, os filtros de maior tamanho de poro e, portanto, mais permeáveis sofrerão um maior dano mecânico sob choque térmico. Já para os filtros de menor tamanho de poro, pode-se assumir que, devido a sua baixa permeabilidade, somente os filamentos da superfície externa do filtro estão submetidos à elevada severidade de choque térmico. Por outro lado, a severidade de choque térmico para os filamentos mais internos passa a ter uma dependência com o tempo de acesso da água até aquele ponto e da temperatura dessa mesma água.

Além da permeabilidade, o tamanho de poro também influencia a área superficial por unidade de volume dos filtros e esta, por sua vez, afeta a troca de calor entre o fluido e o material. Na Fig. 6 apresenta-se o comportamento da área superficial por unidade de volume em função do tamanho de poro dos filtros.

$\mathrm{O}$ aumento da área superficial por unidade de volume, devido à diminuição do tamanho de poro, afeta a troca de calor no fluido

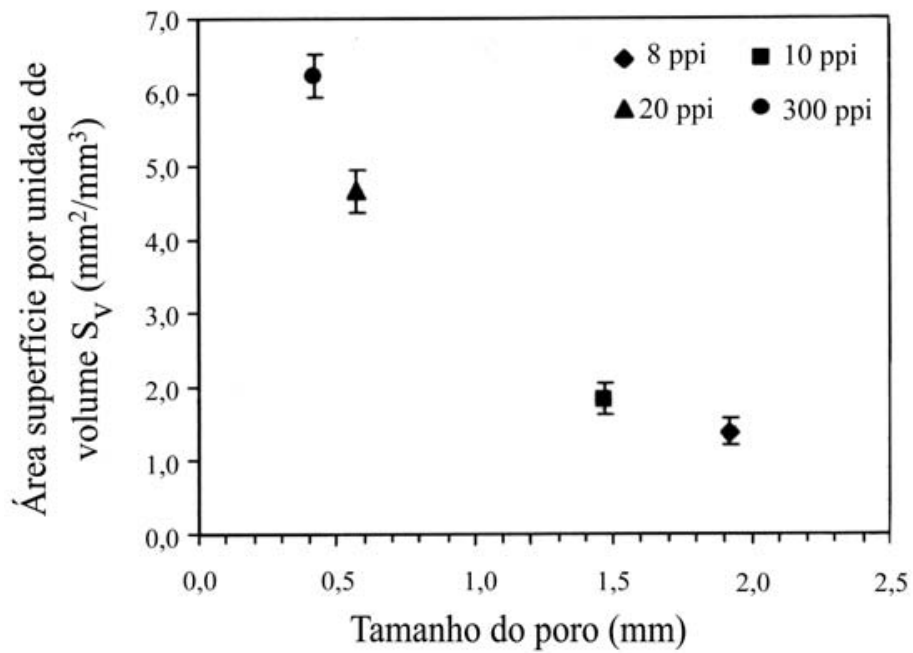

Figura 6: Efeito do tamanho de poro sobre a área superficial por unidade de volume $\left(\mathrm{S}_{\mathrm{v}}\right)$ dos filtros de $8,10,20$ e 30 ppi.

[Figure 6: Effect of pore size on the surface area per unit volume $\left(S_{v}\right)$ of 8 , 10, 20 e 30 ppi filters.]

$\left(\mathrm{T}_{\text {fluido }}\right)$ e entre este e o material sólido $\left(\mathrm{T}_{\text {sólido }}-\mathrm{T}_{\text {fluido }}\right)$. A medida que $\mathrm{a}$ área superficial $\left(\mathrm{S}_{\mathrm{v}}\right)$ aumenta, o fluido passa por um número maior de barreiras sólidas e estas, ao perderem calor por convecção, aquecem o fluido aumentando, portanto, a $\mathrm{T}_{\text {fluido }}$. Decorrente disso, a diferença de temperatura entre o filamento sólido e o fluido em contato com sua superfície $\left(\mathrm{T}_{\text {sólido }}-\mathrm{T}_{\text {fluido }}\right)$ diminui. Consequentemente, esperar-se-ia um menor dano mecânico causado por choque térmico para aqueles filtros de maior área superficial (tamanho de poro menor), considerando apenas este efeito.

O comportamento da condutividade térmica total dos filtros de $\mathrm{Al}_{2} \mathrm{O}_{3}-\mathrm{SiC}$ é ilustrado na Fig. 7 em função do tamanho de poro e da temperatura.

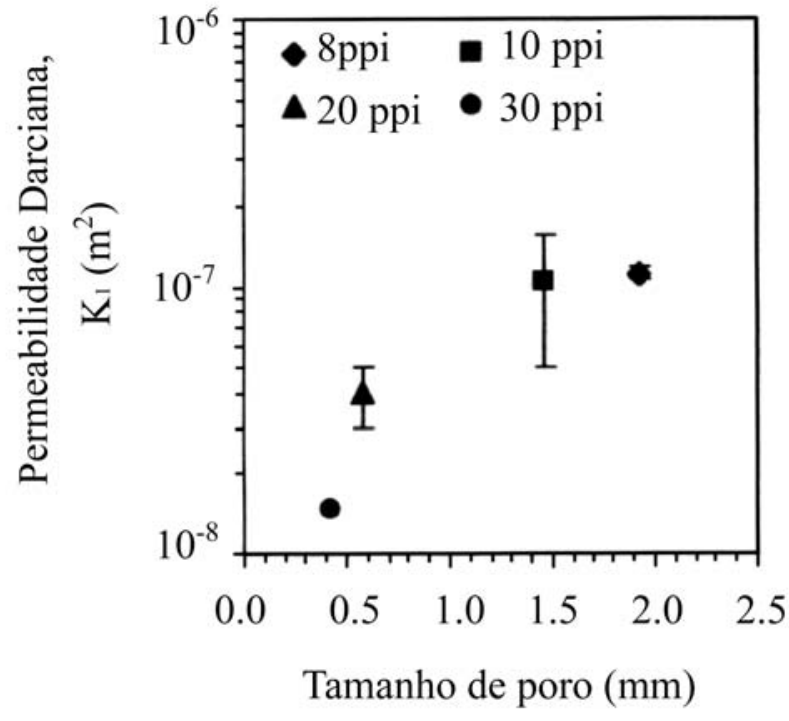

a)

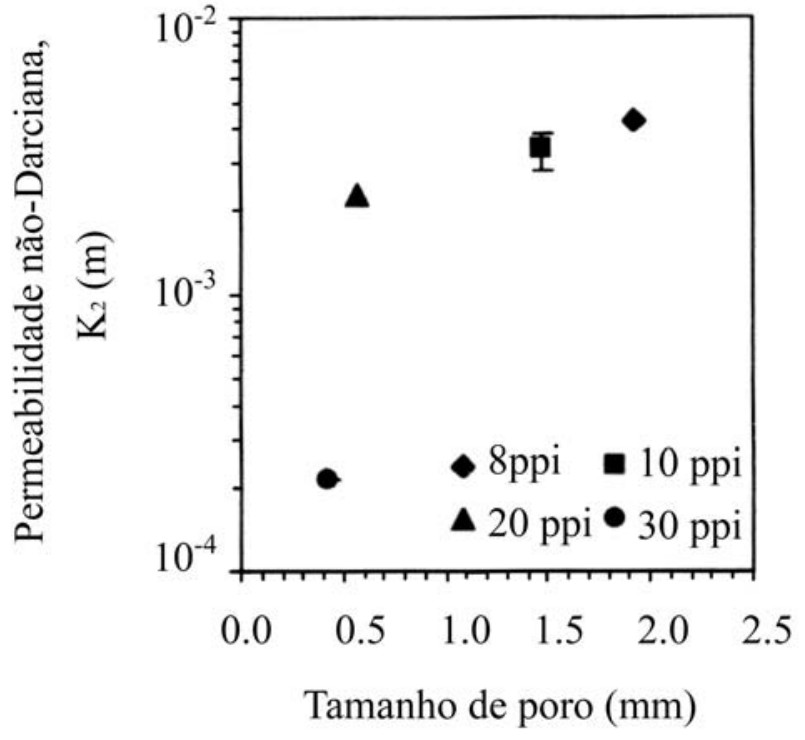

b)

Figura 5: Comportamento das constantes de permeabilidade (a) Darciana, $\mathrm{k}_{1}$, e (b) não-Darciana, $\mathrm{k}_{2}$, em função do tamanho de poro dos filtros de $\mathrm{Al}_{2} \mathrm{O}_{3}-\mathrm{SiC}_{\text {. }}$

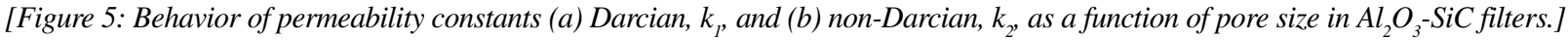




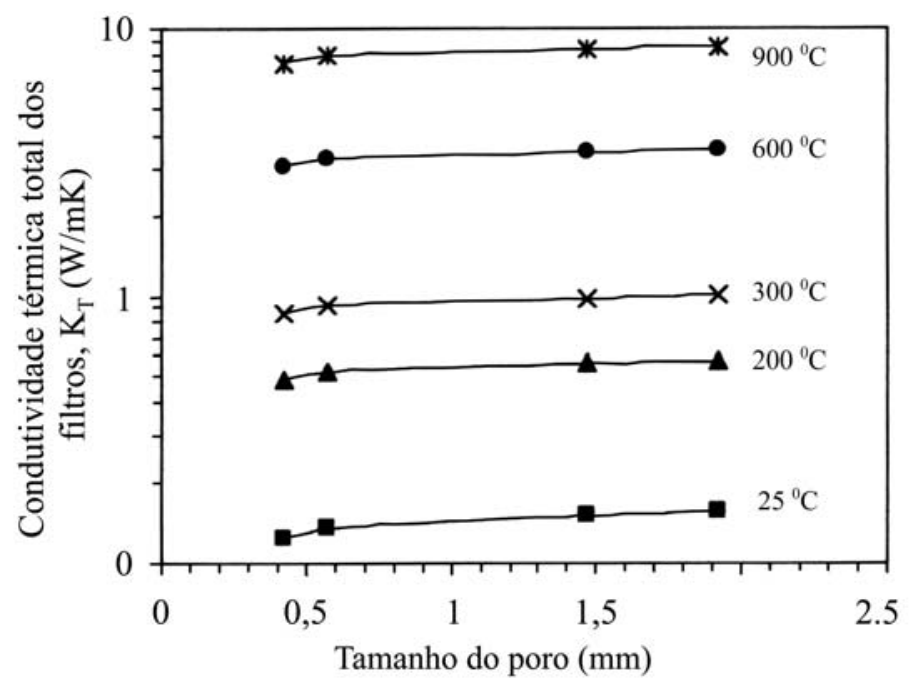

Figura 7: Efeito do tamanho de poro e da temperatura sobre a condutividade térmica total $\left(\mathrm{K}_{\mathrm{T}}\right)$ dos filtros de $\mathrm{Al}_{2} \mathrm{O}_{3}-\mathrm{SiC}$.

[Figure 7: Effect of pore size and temperature on the total thermal conductivity $\left(\mathrm{K}_{\mathrm{T}}\right)$ of $\mathrm{Al}_{2} \mathrm{O}_{3}$-SiC filters.]

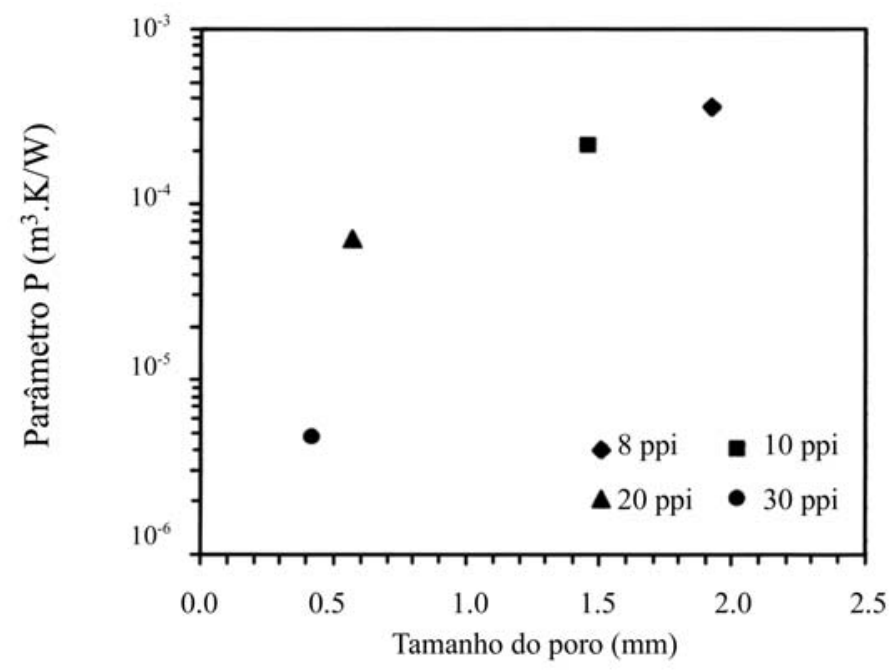

Figura 9: Comportamento do parâmetro $\mathrm{P}$ em função do tamanho de poro dos filtros de 8, 10, 20 e 30 ppi.

[Figure 9: Behavior of parameter $P$ as a function of pore size in 8, 10, 20 and 30 ppi filters.]

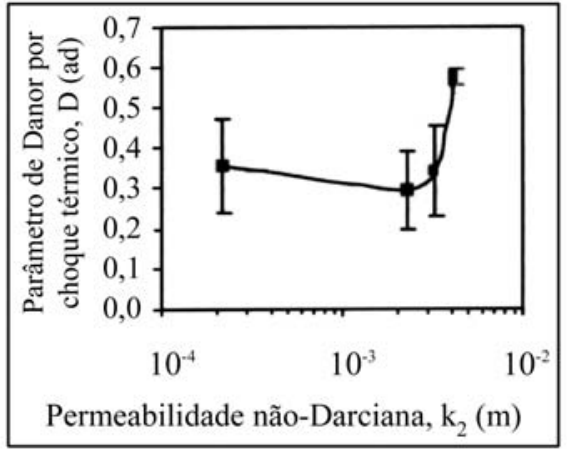

(a)

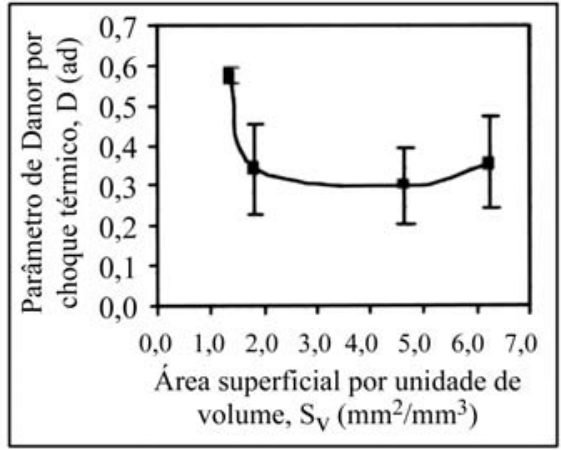

(b)

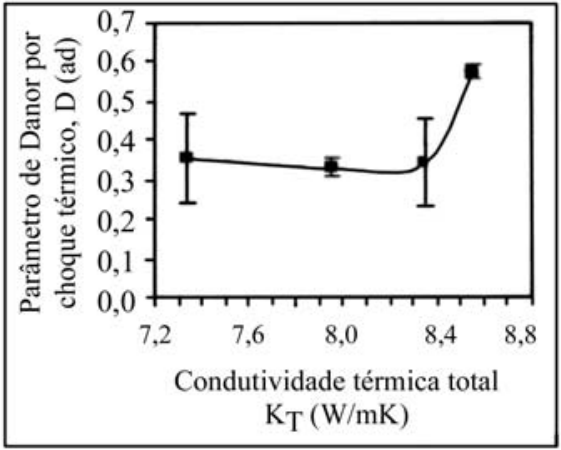

(c)

Figura 8: Comportamento do parâmetro de dano, D, após $\Delta \mathrm{T}$ de $900{ }^{\circ} \mathrm{C}$ em função das propriedades: (a) permeabilidade, $\mathrm{k}_{2}$, (b) área superficial por unidade de volume, $\mathrm{S}_{\mathrm{v}}$ e (c) condutividade térmica total, $\mathrm{K}_{\mathrm{T}}$, dos filtros.

[Figure 8: Damage parameter behavior after $\Delta T$ of $900^{\circ} \mathrm{C}$ as a function of filters properties: (a) permeability, $k_{2}$, (b) surface area per unit volume, $S_{v}$ and (c) total thermal conductivity, $\left.K_{T}\right]$

Nota-se na Fig. 7 que, para uma mesma temperatura, a condutividade térmica aumenta com o aumento do tamanho de poro do filtro, devido a uma menor área superficial específica $\left(\mathrm{S}_{\mathrm{v}}\right)$ que contribui para um menor espalhamento do fluxo de calor. Já o aumento da condutividade térmica em função da temperatura é decorrente da superior contribuição da radiação de calor no cálculo da condutividade térmica total $[2,12]$.

Ao se correlacionar isoladamente estas três propriedades dos filtros (permeabilidade, área superficial e condutividade térmica) com o parâmetro de dano (D) por choque térmico, não se verifica uma tendência clara, conforme ilustrado na Fig. 8 .

Na Fig. 8 foram consideradas a constante de permeabilidade não-Darciana $\left(\mathrm{k}_{2}\right)$ e a condutividade térmica total dos filtros $\left(\mathrm{K}_{\mathrm{T}}\right)$ calculada a $900{ }^{\circ} \mathrm{C}$, por corresponder, respectivamente, à permeabilidade dos filtros em altas velocidades de escoamento e à máxima variação de temperatura sofrida pelos filtros estudados.

Uma vez que o módulo de Biot $(\beta)$ é um indicativo da severidade do choque térmico a que um material é submetido e que, de acordo com a equação (D), para cálculo de $\beta$, o tamanho característico $\left(\mathrm{r}_{\mathrm{m}}\right)$ pode ser representado pela razão do volume pela área superficial $\left(\mathrm{V} / \mathrm{S}_{\mathrm{v}}\right)$ e o coeficiente de transferência de calor $(\mathrm{h})$ depende da permeabilidade do material, pode-se supor que o módulo de Biot $(\beta)$ possa ser indiretamente expresso por:

$$
\beta \infty \frac{\text { permeabilidade }}{\text { área superficial } \mathrm{x} \text { condutividade térmica }}
$$

Assim, uma possível combinação entre estas propriedades é sugerida neste trabalho como o parâmetro $\mathrm{P}$, dado por:

$$
\mathrm{P} \infty \cdot \frac{\mathrm{k}_{2}}{\mathrm{~S}_{\mathrm{v}} \cdot \mathrm{K}_{\mathrm{T}}}
$$




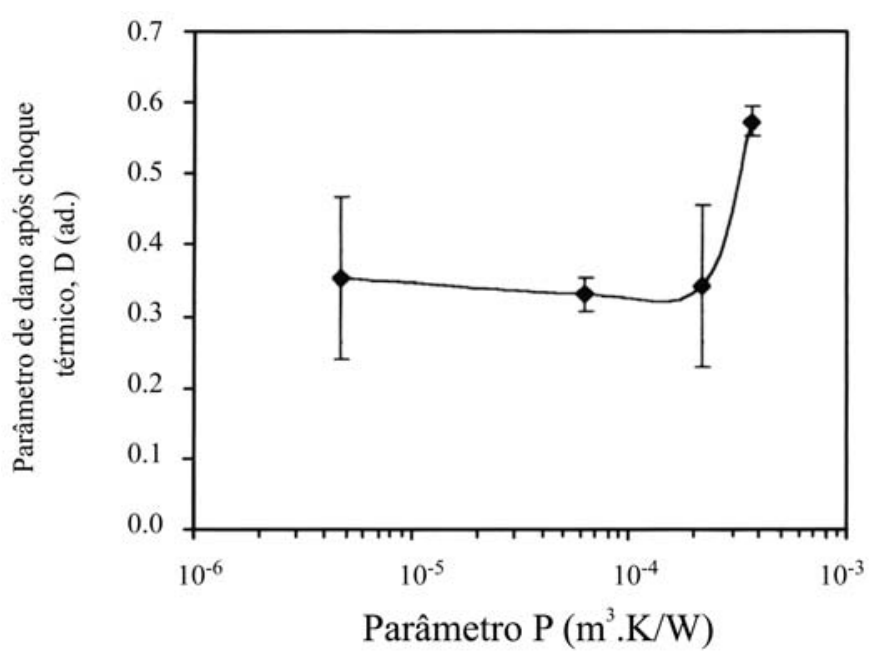

Figura 10: Comportamento do parâmetro de dano, D, em função do parâmetro $\mathrm{P}$ para os filtros de $\mathrm{Al}_{2} \mathrm{O}_{3}-\mathrm{SiC}$ de 8, 10, 20 e 30 ppi.

[Figure 10: Damage parameter behavior as a function of $P$ parameter for 8, 10, 20 and $30 \mathrm{ppi}_{2} \mathrm{O}_{3}$-SiC filters.]

O gráfico da Fig. 9 ilustra o comportamento do parâmetro P em função do tamanho de poro dos filtros de $\mathrm{Al}_{2} \mathrm{O}_{3}-\mathrm{SiC}$ de 8, 10, 20 e 30 ppi.

A priori pode-se supor que, de acordo com a Fig. 9, o aumento do tamanho de poro do filtro conduz a uma maior severidade do choque térmico, representada no gráfico pelo parâmetro $\mathrm{P}$ proposto neste trabalho.

Mas, se o dano mecânico causado por choque térmico nos filtros estudados dependesse unicamente da permeabilidade, área superficial e condutividade térmica destes materiais, a correlação gráfica entre o parâmetro $\mathrm{P}$ proposto e o de dano por choque térmico, $\mathrm{D}$, deveria ser de proporcionalidade, o que não é observado na Fig. 10.

Contudo, embora a relação entre as propriedades de permeabilidade, área superficial por unidade de volume e condutividade térmica não seja, por si só, suficiente para explicar o comportamento do dano mecânico por choque térmico nos filtros cerâmicos estudados, acredita-se que estas propriedades associadas à tensão de fratura $\left(\sigma_{\mathrm{F}}\right)$, ao módulo elástico $(\mathrm{E})$ e à energia de fratura $\left(\gamma_{\mathrm{WOF}}\right)$ dos filtros, possam contribuir para a elaboração de um modelo teórico que expresse o dano verificado experimentalmente.

\section{CONCLUSÕES}

As principais conclusões deste trabalho são:

1. O choque térmico em filtros cerâmicos está relacionado à tensão térmica que atua nos filamentos individualmente, porém o dano mecânico está associado à somatória dos efeitos verificados no filtro como um todo.

2. O parâmetro de dano por choque térmico, D, nos filtros estudados apresentou uma clara dependência com a severidade do choque $(\Delta \mathrm{T})$. Porém, a correlação do dano com o tamanho de poro indicou apenas um valor diferenciado para a amostra de filtro de 8 ppi.

3. O tamanho de poro (célula) influenciou a permeabilidade, a área superficial por unidade de volume e a condutividade térmica dos filtros. Contudo, ao se correlacionar estas propriedades com o parâmetro de dano por choque térmico, D, não se verificou uma tendência clara.

4. Embora o parâmetro $P$ proposto neste trabalho não seja, por si só, suficiente para explicar o comportamento do dano por choque térmico nos filtros cerâmicos estudados, acredita-se que na elaboração de um modelo de dano para esta classe de materiais cerâmicos este parâmetro ou as propriedades que o constituem devem ser consideradas.

\section{AGRADECIMENTOS}

Os autores agradem à FAPESP, ao engenheiro Jean Yamamoto pelo auxílio dado para a realização deste trabalho e ao Prof. Dr. José de Anchieta Rodrigues pela cuidadosa revisão do texto.

\section{REFERÊNCIAS}

[1] V. R. Salvani, Dissertação de Mestrado, DEMa, UFSCar (1995).

[2] L. J. Gibson, M. F. Ashby, Cellular Solids-Structure and Properties, $2^{\text {nd }}$ Ed., Pergamon Press, N. York (1998).

[3] R. M. Orenstein, J. D. Green, J. Am. Ceram. Soc. 75, 7 (1992) 1899-1905.

[4] V. R. Vedula, D. J. Green, J. R. Hellman, J. Am. Ceram. Soc. 82, 3 (1999) 649-656.

[5] W. D. Kingery, H. K. Bowen, D. R. Uhlmann, Introduction to Ceramics, $2^{\text {nd }}$ Ed., Wiley, N. York (1976).

[6] H. Wang, R. N. Singh, Intern. Mat. Rev. 39, 6 (1994) 228-244. [7] P. F. Becher, D. Lewis III, K. R. Carman, A. C. Gonzalez, Am. Ceram. Soc. Bull. 59, 5 (1980) 542-548.

[8] J. Saggio-Woyansky, C. E. Scott, Am. Ceram. Soc. Bull. 71, 11 (1992) 1674-1682.

[9] V. R. Salvini, M. D. M. Innocentini, V. C. Pandolfelli, Am. Ceram. Soc. Bull. 79, 5 (2000) 49-54.

[10] J. T. Richardson, Y. Peng, D. Remue, Appl. Catal. A 204 (2000) 19-32.

[11] M. D. M. Innocentini, V. R. Salvini, J. R. Coury, V. C. Pandolfelli, Am. Ceram. Soc. Bull. 78, 9 (1999) 78-84.

[12] L. R. Glicksman, M. R. Torpey, Proc. Polyurethanes World Cong. (1987) 80-84.

[13] D. D. Brown, D. J. Green, J. Am. Ceram. Soc. 77, 6 (1994) 1467-1472.

(Rec. 22/05/01, Ac. 22/12/01) 